\title{
Successful treatment of suspected organizing pneumonia in a patient with Middle East respiratory syndrome coronavirus infection: a case report
}

\author{
Insu Kim, Jeong Eun Lee, Kye-Hyung Kim, Shinwon Lee, Kwangha Lee, Jeong Ha Mok \\ Department of Internal Medicine, Pusan National University School of Medicine, Busan, Korea \\ Correspondence to: Jeong Ha Mok. Department of Internal Medicine, Pusan National University Hospital, 179 Gudeok-ro, Seo-gu, Busan, 602-739, \\ Korea. Email: mokgamokga@gmail.com.
}

\begin{abstract}
A 54-year-old man with Middle East respiratory syndrome coronavirus (MERS-CoV) infection was transferred to our hospital. We initiated anti-viral drugs and supportive care. The patient's fever and chills disappeared 3 days after admission and the results of serial follow-up reverse transcription-polymerase chain reaction testing for MERS-CoV was negative soon thereafter. He was discharged from the hospital 14 days after admission with no symptoms; however, he presented with a fever 7 days after discharge and was re-hospitalized. Chest radiographs showed newly developed consolidative opacity. His fever persisted for 3 days after commencing empirical antibiotics. Subsequent contrast-enhanced computed tomography (CT) of the chest showed focal patchy airspace consolidation and ground-glass opacities (GGOs) in a subpleural lesion of the right lower and left upper lobes, which was indicative of organizing pneumonia. We initiated empirical corticosteroid treatment for this illness, and his fever markedly subsided 1 day later. A chest radiograph showed improvement in the lung lesions, and he was discharged from the hospital 10 days after re-admission. The corticosteroid dose was gradually tapered over 2 months at the outpatient clinic, and a follow-up CT scan showed complete resolution of the consolidation and GGOs.
\end{abstract}

Keywords: Corticosteroid; Middle East respiratory syndrome coronavirus (MERS-CoV); organizing pneumonia

Submitted May 26, 2016. Accepted for publication Jul 25, 2016.

doi: $10.21037 /$ jtd.2016.09.26

View this article at: http://dx.doi.org/10.21037/jtd.2016.09.26

\section{Introduction}

Organizing pneumonia is a type of lung disease characterized by proliferation of granulation tissue in the alveoli or alveolar duct, with or without obliteration of distal bronchioles (1). In addition to the cryptogenic form, it can develop secondarily for many reasons, such as due to virus infections (1).

There are several reports of organizing pneumonia after coronavirus infections, especially severe acute respiratory syndrome coronavirus (SARS-CoV) infection (2-5). However, there is limited information on lung disease caused by Middle East respiratory syndrome coronavirus (MERS-CoV) infection. In addition, few studies have reported cases of organizing pneumonia associated with
MERS-CoV infection. Here, we report a case of suspected organizing pneumonia after MERS-CoV infection, which was successfully treated with a corticosteroid.

\section{Case presentation}

A 54-year-old man with MERS-CoV infection was transferred to our hospital for treatment in an airborneinfection isolation room. He had a history of hypertension, diabetes, hepatitis B infection, and liver cirrhosis. He had been admitted to another hospital for acute exacerbation of hepatitis B and liver cirrhosis for the past month. During that hospital stay, one patient who shared the room was diagnosed with MERS-CoV infection (MERS outbreak in Korea, 2015). Our patient presented with fever and 


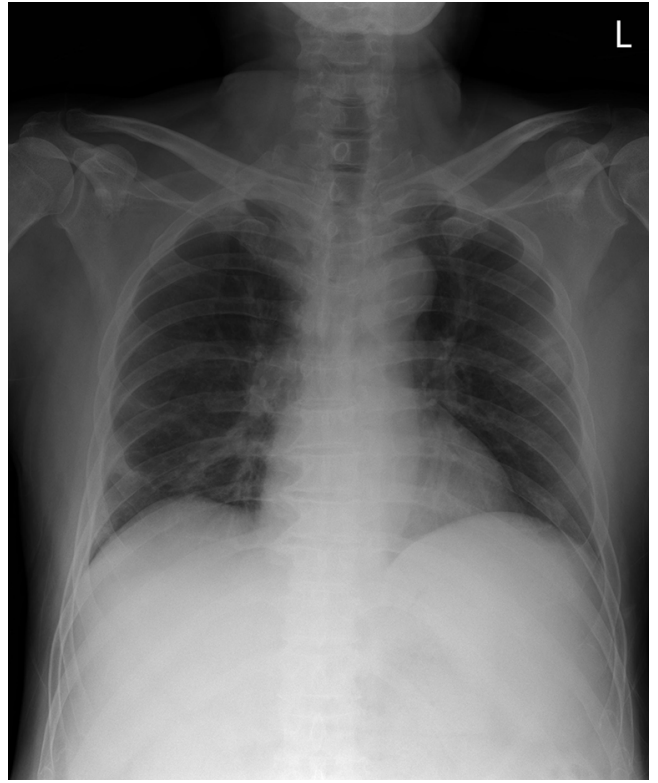

Figure 1 Consolidative opacities in the right lower and left upper lung zones.

chills 5 days later, and reverse transcription-polymerase chain reaction (RT-PCR) analysis of nasopharyngeal swab specimens confirmed that he had developed a MERS-CoV infection.

Initial vital signs at our hospital were: blood pressure, 128/91 $\mathrm{mmHg}$; heart rate, 109/min; respiratory rate, 24/min; and temperature, $37.0^{\circ} \mathrm{C}$. Normal breathing and heart sounds were detected on a physical examination. Laboratory data on admission were as follows: white blood cell count, $6,520 / \mu \mathrm{L}$ (neutrophils, $52.8 \%$; lymphocytes, $31.7 \%$; and eosinophils, $1.2 \%$ ); hemoglobin, $11.1 \mathrm{~g} / \mathrm{dL}$; platelet count, $115,000 / \mu \mathrm{L}$; international normalized ratio of prothrombin time (PT-INR), 1.52; aspartate aminotransferase (AST), $165 \mathrm{IU} / \mathrm{L}$; alanine aminotransferase (ALT), $79 \mathrm{IU} / \mathrm{L}$; total bilirubin, $11.76 \mathrm{mg} / \mathrm{dL}$; direct bilirubin, $8.85 \mathrm{mg} / \mathrm{dL}$; total protein, $6.4 \mathrm{~g} / \mathrm{dL}$; albumin, $2.2 \mathrm{~g} / \mathrm{dL}$; and C-reactive protein $(\mathrm{CRP}), 1.24 \mathrm{mg} / \mathrm{dL}$. Chest radiographic findings were normal.

One day after admission to our hospital, we initiated oral ribavirin $(2,000 \mathrm{mg}$ loading, then $1,200 \mathrm{mg}$ three times/day) and lopinavir/ritonavir (400/100 mg two times/day) to treat the MERS-CoV infection. However, ribavirin and lopinavir/ritonavir were stopped after 5 days of treatment because he presented with hemolytic anemia and thrombocytopenia (hemoglobin, $10.2 \mathrm{~g} / \mathrm{dL}$; platelet count, $89,000 / \mu \mathrm{L}$; and lactate dehydrogenase, $402 \mathrm{IU} / \mathrm{L}$ ), which was suspected to be an adverse drug reaction. Fever and chills disappeared 3 days after admission. The results of serial follow-up RT-PCR testing for MERS-CoV converted to negative soon thereafter. He was discharged from the hospital 14 days after admission with no symptoms. Chest radiographs on the day of discharge were normal.

He presented again with a fever 7 days after discharge and was re-hospitalized. He had no other symptoms except chills. Vital signs were normal except temperature $\left(38.1^{\circ} \mathrm{C}\right)$. Laboratory data on re-admission were: white blood cell count, $2,800 / \mu \mathrm{L}$ (neutrophils, $47.1 \%$; lymphocytes, $39.6 \%$; and eosinophils, $0.4 \%$ ); hemoglobin, $10.6 \mathrm{~g} / \mathrm{dL}$; platelet

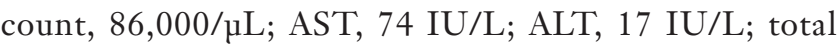
bilirubin, $4.33 \mathrm{mg} / \mathrm{dL}$; and CRP, $1.15 \mathrm{mg} / \mathrm{dL}$. RT-PCR for MERS-CoV was negative. However, chest radiographs showed newly developed consolidative opacity in the right lower and left upper lung zones (Figure 1). Neither bacteria nor fungus were detected on a sputum examination. Blood antibody and urine antigen tests for several bacteria were negative (Streptococcus pneumoniae, Mycoplasma pneumoniae, Chlamydia pneumoniae, and Legionella). Antigen tests for several viruses were also negative (influenza virus, parainfluenza virus, adenovirus, etc.). An empirical antibiotic (cefotaxime and levofloxacin) treatment was started; however, the fever was persistent 3 days after commencing the antibiotics. Subsequently, a contrast-enhanced chest computed tomography (CT) scan was performed. Focal patchy air-space consolidation and ground-glass opacities (GGOs) were noted in a subpleural lesion of the right lower and left upper lobes, which was compatible with organizing pneumonia (Figure 2).

Because he had a bleeding tendency due to liver cirrhosis (platelet count, 69,000/ $\mu \mathrm{L}$; PT-INR, 1.56) and we were concerned about the MERS-CoV contagion, although the MERS-CoV RT-PCR test was negative, we initiated empirical corticosteroid (intravenous $32 \mathrm{mg}$ methylprednisolone) treatment for the suspected organizing pneumonia, beginning on day 4 of re-admission, instead of taking a lung biopsy or performing bronchoalveolar lavage. His fever subsided markedly 1 day after starting the methylprednisolone. A chest radiograph showed improvement in the lung lesions, and he was discharged from the hospital 10 days after re-admission with oral methylprednisolone $(28 \mathrm{mg})$. The methylprednisolone was tapered gradually over 2 months at the outpatient clinic. A follow-up low-dose chest CT scan taken 1 month after methylprednisolone was discontinued showed complete resolution of the consolidation and GGOs (Figure 3, Table 1). 

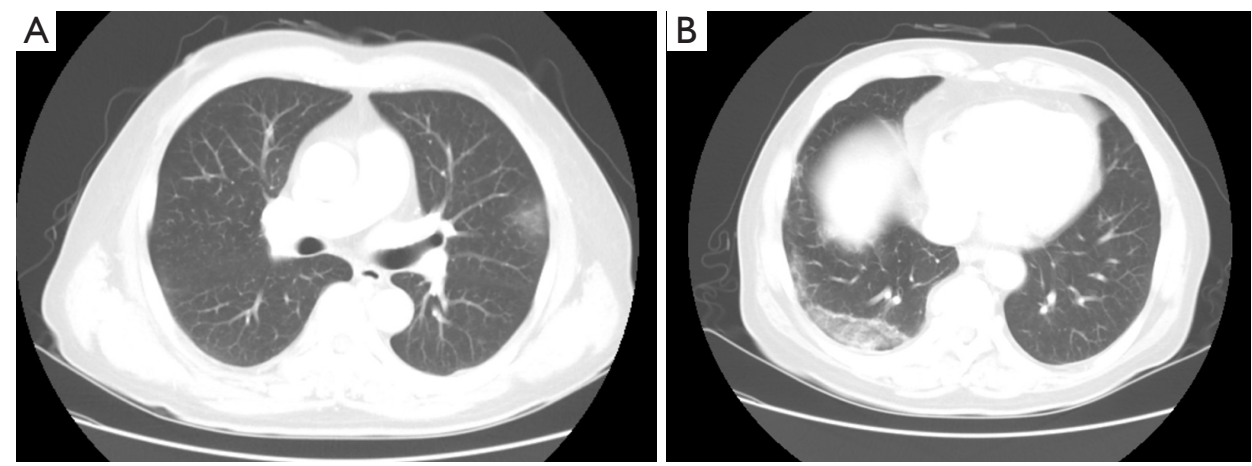

Figure 2 Contrast-enhanced chest computed tomography scan performed 3 days after re-admission. (A) Ground-glass opacities in a subpleural lesion of the left upper lung lobe; (B) patchy air-space consolidation and ground-glass opacities in a subpleural lesion of the right lower lung lobe.

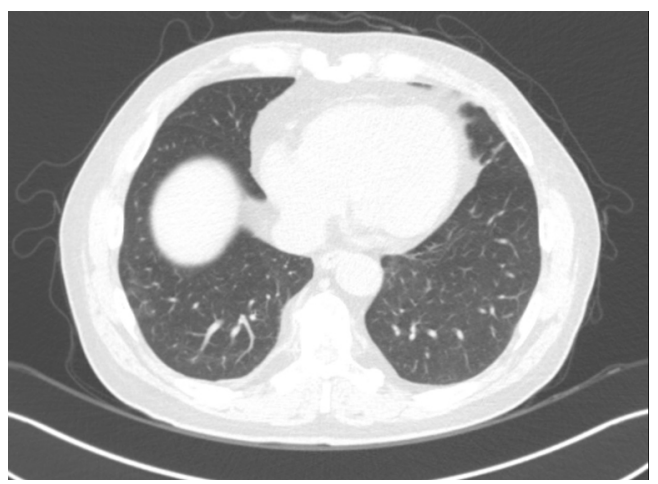

Figure 3 Complete resolution of consolidation and ground-glass opacities after the corticosteroid treatment.

\section{Discussion}

Organizing pneumonia is divided into an idiopathic form [cryptogenic organizing pneumonia, previously called bronchiolitis obliterans organizing pneumonia (BOOP)] and a secondary form. Secondary organizing pneumonia can result from infections (including bacteria, viruses, parasites, and fungi), connective tissue disease, hematological disorders or malignancies, use of certain drugs, and radiation treatment (1). Corticosteroids are the mainstay for treating severe cases of organizing pneumonia, and often result in rapid clinical and radiological improvements (1).

Virus infections are not an uncommon cause of secondary organizing pneumonia. Several cases of organizing pneumonia after influenza A virus infection have been reported $(6,7)$. In addition, adenovirus, cytomegalovirus, human immunodeficiency virus, and respiratory syncytial virus have been reported to be possible causes of organizing pneumonia. With regard to the coronavirus, several studies have reported organizing pneumonia after SARS$\mathrm{CoV}$ infections and a study has reported case of suggestive organizing pneumonia associated with MERS-CoV infection $(2-5,8)$. However, currently there are no largescale epidemiologic data of this illness after coronavirus infection that includes incidence, clinical course, treatment, or clinical outcomes.

MERS is a viral respiratory disease caused by the novel coronavirus (MERS-CoV) (9). Over 1,700 laboratoryconfirmed cases from 27 countries were detected, and approximately $36 \%$ of patients have died due to a MERS-CoV infection since 2012 (10). Previous studies have indicated that men have a higher infection rate of MERS-CoV compared to women, with a median age of 56-62 years $(11,12)$. The majority of patients were infected by person-to-person transmission in healthcare facilities or households (11-13), with a median incubation period of 5.2 days (12). Fever, cough, and shortness of breath were the most common symptoms. Most fatal cases were frequently associated with significant comorbidities such as obesity, renal disease, cardiac disease, diabetes mellitus, lung disease, and immunosuppressive conditions. In these cases, almost all of the patients developed pneumonia and more than $70 \%$ required admission to the intensive care unit, requiring ventilator care (11-13).

The radiological features of MERS are even less clearly identified. Several studies have reported that the radiologic features of MERS are usually non-specific and are similar to those of pneumonia from other causes (13-15). These radiological features may progress from mild unilateral focal lesions to acute respiratory distress syndrome and extensive 
Table 1 Summary of patient's clinical course

\begin{tabular}{|c|c|}
\hline $\begin{array}{l}\text { Days after initial } \\
\text { diagnosis }\end{array}$ & Clinical events \\
\hline 0 & Admission \\
\hline 1 & Ribavirin, lopinavir/ritonavir treatment \\
\hline \multirow[t]{2}{*}{3} & Fever disappearance \\
\hline & Negative conversion of RT-PCR for MERS-CoV \\
\hline 5 & $\begin{array}{l}\text { Ribavirin, lopinavir/ritonavir stop due to adverse } \\
\text { drug reaction }\end{array}$ \\
\hline 14 & $\begin{array}{l}\text { Discharge with no symptom } \\
\text { Normal chest radiographs }\end{array}$ \\
\hline \multirow[t]{3}{*}{21} & Re-admission due to fever \\
\hline & $\begin{array}{l}\text { Newly developed consolidative opacity on chest } \\
\text { radiographs }\end{array}$ \\
\hline & Empirical antibiotic treatment \\
\hline 23 & Suspected organizing pneumonia on chest CT \\
\hline 24 & Intravenous methylprednisolone $32 \mathrm{mg}$ \\
\hline \multirow[t]{2}{*}{25} & Fever disappearance \\
\hline & Improvement of chest radiographs \\
\hline 30 & Discharge (oral methylprednisolone 28 mg) \\
\hline $30-82$ & $\begin{array}{l}\text { Gradual tapering of methylprednisolone in } \\
\text { outpatient clinic }\end{array}$ \\
\hline 110 & Complete resolution on follow-up chest CT \\
\hline
\end{tabular}

$\mathrm{CT}$, computed tomography; MERS-CoV, Middle East respiratory syndrome coronavirus; RT-PCR, reverse transcriptionpolymerase chain reaction.

multifocal or bilateral involvement in severe cases. However, another study reported that some patients with MERS$\mathrm{CoV}$ infection have a predominance of patchy air-space opacities and more extensive GGOs than consolidations in the subpleural lung, and often in the lower lung zone on a CT scan, suggestive of an organizing pneumonia (8). Several animal model studies have reported that organizing pneumonia is not uncommon in animals with a MERS$\mathrm{CoV}$ infection $(16,17)$. Although we could not pathologically confirm organizing pneumonia due to the patient's bleeding tendency, his lung lesions probably reflected organizing pneumonia because a marked clinical and radiological response was achieved soon after commencing the corticosteroid treatment. In addition, CT imaging features of organizing pneumonia are characteristic and the diagnostic accuracy of CT for organizing pneumonia is high (79\%) (18).
Our patient presented with suspected organizing pneumonia 3 weeks after initial diagnosis of MERS-CoV infection, which is similar in duration to other cases of this illness after coronavirus infections $(3-5,8)$. However, his clinical course differed from that of previously reported MERS-CoV-infected patients, in that the latter group presented with abnormalities, including an organizing pneumonia pattern on chest radiographs or CT scan as their clinical course worsened, whereas the patient in this study presented with an organizing pneumonia pattern on a chest CT scan 1 week after complete symptom resolution and negative conversion of MERS-CoV detected by RT-PCR.

To date, there is no established pathogenesis of organizing pneumonia after MERS-CoV infection, although one study suggested that viral-type organizing pneumonia may result from immune system stimulation by viral antigens (19). Inhaled viral antigens stimulate inflammation and lymphokine production in bronchus-associated lymphoid tissue (BALT), and variation in a patient's immune system determine the response to injury towards a specific type of bronchiolitis. However, the evidence for this theory is insufficient; thus additional studies are required to identify the precise mechanism underlying organizing pneumonia after MERS-CoV infection.

This study had some limitations. We could not completely rule out other possible causes of organizing pneumonia, particular drug use. Ribavirin has been reported as a possible cause of organizing pneumonia (20). However, the duration of ribavirin use by our patient was too short to cause organizing pneumonia, as organizing pneumonia usually develops after 10 weeks of ribavirin use (20).

\section{Conclusions}

In conclusion, the present case suggests that organizing pneumonia can occur in a patient with a MERS-CoV infection. Furthermore, corticosteroid use may be helpful for treating suspected organizing pneumonia in a patient with MERS-CoV infection.

\section{Acknowledgements}

This work was supported by clinical research grant from Pusan National University Hospital in 2015.

\section{Footnote}

Conflicts of Interest: The authors have no conflicts of interest 
to declare.

Informed Consent: Written informed consent was obtained from the patient for publication of this manuscript and any accompanying images.

\section{References}

1. Cordier JF. Cryptogenic organising pneumonia. Eur Respir J 2006;28:422-46.

2. Tse GM, To KF, Chan PK, et al. Pulmonary pathological features in coronavirus associated severe acute respiratory syndrome (SARS). J Clin Pathol 2004;57:260-5.

3. Hsiao CH, Wu MZ, Chen CL, et al. Evolution of pulmonary pathology in severe acute respiratory syndrome. J Formos Med Assoc 2005;104:75-81.

4. Hwang DM, Chamberlain DW, Poutanen SM, et al. Pulmonary pathology of severe acute respiratory syndrome in Toronto. Mod Pathol 2005;18:1-10.

5. Lai RQ, Feng XD, Gu YY, et al. Pathological changes of lungs in patients with severity acute respiratory syndrome. Zhonghua Bing Li Xue Za Zhi 2004;33:354-7.

6. Ajlan AM, Quiney B, Nicolaou S, et al. Swine-origin influenza A (H1N1) viral infection: radiographic and CT findings. AJR Am J Roentgenol 2009;193:1494-9.

7. Gill JR, Sheng ZM, Ely SF, et al. Pulmonary pathologic findings of fatal 2009 pandemic influenza A/H1N1 viral infections. Arch Pathol Lab Med 2010;134:235-43.

8. Ajlan AM, Ahyad RA, Jamjoom LG, et al. Middle East respiratory syndrome coronavirus (MERS-CoV) infection: chest CT findings. AJR Am J Roentgenol 2014;203:782-7.

9. Zaki AM, van Boheemen S, Bestebroer TM, et al. Isolation of a novel coronavirus from a man with pneumonia in Saudi Arabia. N Engl J Med 2012;367:1814-20.

10. World Health Organization. Fact sheet on MERSCoV, June, 2015. Available online: http://www.who.int/

Cite this article as: Kim I, Lee JE, Kim KH, Lee S, Lee $\mathrm{K}$, Mok JH. Successful treatment of suspected organizing pneumonia in a patient with Middle East respiratory syndrome coronavirus infection: a case report. J Thorac Dis 2016;8(10):E1190-E1194. doi: 10.21037/jtd.2016.09.26 mediacentre/factsheets/mers-cov/en/

11. Saad M, Omrani AS, Baig K, et al. Clinical aspects and outcomes of 70 patients with Middle East respiratory syndrome coronavirus infection: a single-center experience in Saudi Arabia. Int J Infect Dis 2014;29:301-6.

12. Assiri A, McGeer A, Perl TM, et al. Hospital outbreak of Middle East respiratory syndrome coronavirus. N Engl J Med 2013;369:407-16.

13. Assiri A, Al-Tawfiq JA, Al-Rabeeah AA, et al. Epidemiological, demographic, and clinical characteristics of 47 cases of Middle East respiratory syndrome coronavirus disease from Saudi Arabia: a descriptive study. Lancet Infect Dis 2013;13:752-61.

14. Chan JF, Lau SK, To KK, et al. Middle East respiratory syndrome coronavirus: another zoonotic betacoronavirus causing SARS-like disease. Clin Microbiol Rev 2015;28:465-522.

15. Arabi YM, Arifi AA, Balkhy HH, et al. Clinical course and outcomes of critically ill patients with Middle East respiratory syndrome coronavirus infection. Ann Intern Med 2014;160:389-97.

16. Munster VJ, de Wit E, Feldmann H. Pneumonia from human coronavirus in a macaque model. $\mathrm{N}$ Engl J Med 2013;368:1560-2.

17. Yao Y, Bao L, Deng W, et al. An animal model of MERS produced by infection of rhesus macaques with MERS coronavirus. J Infect Dis 2014;209:236-42.

18. Johkoh T, Müller NL, Cartier Y, et al. Idiopathic interstitial pneumonias: diagnostic accuracy of thin-section CT in 129 patients. Radiology 1999;211:555-60.

19. Schlesinger C, Koss MN. The organizing pneumonias: an update and review. Curr Opin Pulm Med 2005;11:422-30.

20. Kumar KS, Russo MW, Borczuk AC, et al. Significant pulmonary toxicity associated with interferon and ribavirin therapy for hepatitis C. Am J Gastroenterol 2002;97:2432-40. 